\title{
Early decreased plasma levels of factor B and C5a are important biomarkers in children with Kawasaki disease
}

\author{
Qing-Mei Zou' ${ }^{1}$ Xiao-Hui Li', Rui-Xia Song ${ }^{1}$, Nan-Ping Xu², Ting Zhang ${ }^{3}$, Ming-Ming Zhang ${ }^{1}$, Yao Lin ${ }^{1}$, Lin Shi', \\ Jin $\mathrm{Fu}^{4}$ and Xiao-Dai Cui ${ }^{4}$
}

\begin{abstract}
BACKGROUND: The mechanisms underpinning Kawasaki disease (KD) are incompletely understood. There is an unmet need for specific biomarkers for the early diagnosis of KD.

METHODS: Eighty-five KD patients suffering from acute-phase and subacute-phase KD, 40 healthy children, and 40 febrile children comprised the study cohort. An enzyme-linked immunosorbent assay was used to measure plasma levels of $\mathrm{C} 1 \mathrm{q}$, Clq-circulating immune complex (C1q-CIC), mannan-binding lectin-associated serine protease (MASP)-1, factor B, C4d, C3d, C5a, C5b-9 and CD59.
\end{abstract}

RESULTS: Plasma concentrations of factor B and $\mathrm{C} 5 \mathrm{a}$ in the acute phase were lower than those in healthy and febrile control groups (all $P<0.05$ ). Compared with acute-phase KD patients, plasma concentrations of C1q, factor B, and C3d in KD patients were increased significantly $(P<0.05)$, but those of C4d, MASP-1 and CD59 decreased significantly $(P<0.05)$, in patients with sub-acute KD.

CONCLUSION: These data suggest that more than one pathway in the complement system is activated in KD. Importantly, decreased plasma concentrations of factor $B$ and $C 5 a$ in the acute phase (6-10 d) could be employed as biomarkers for the early diagnosis of $\mathrm{KD}$.

$K^{2}$ awasaki disease $(\mathrm{KD})$ is an acute, self-limiting, nonspecific systemic vascular inflammatory syndrome. KD is considered to be caused by infectious agents in genetically susceptible children. The diagnosis of KD is dependent upon clinical manifestations; definitive diagnostic markers are lacking. The pathogenesis of $\mathrm{KD}$ is characterized by immune injury to endothelial cells (1) that results in systemic vasculitis or aneurysms in coronary arteries (2).

Vascular endothelial cells are a source of complement and complement regulatory factors. Activated complement can form complement fragments (e.g., C3, C4d, Bb) and the membrane attack complex (MAC) on the cell surface (3). The MAC can mediate the inflammatory response by killing anucleate cells and bacteria, as well as causing cells to secrete a large number of cytokines and growth factors (4). Animal studies have shown that coronary atherosclerotic lesions appear not only during complement activation, but also during complement deposition, which results in tissue injury (5). It also has been demonstrated that complement components are accumulated in cryoglobulinemic vasculitis (6). Other studies have reported dysfunction of vascular endothelial cells (7) and the classical pathway of complement activation in all three layers of aneurysms (8).

With the evidence stated above, we speculated that activation of the complement system might be involved in the pathogenesis of $\mathrm{KD}$, and that activated complement factors could be employed as early diagnostic markers for KD. To test this hypothesis, we investigated plasma levels of various complement components in children with acute KD and subacute KD.

\section{RESULTS}

Changes in Plasma Concentrations of Complement Factors

C1q and C1q-circulating immune complex (C1q-CIC). C1q is a factor of the classical pathway of complement activation. The plasma level of C1q was significantly lower in the KD group than that of the healthy control group $(61.71 \pm 29.02 \mathrm{vs}$. $78.50 \pm 51.13 \mu \mathrm{g} / \mathrm{ml}, P<0.05)$. However, there was no significant difference in the plasma concentration of $\mathrm{C} 1 \mathrm{q}$ between the KD group and febrile control group $(61.71 \pm 29.02$ vs. $56.17 \pm 30.74 \mu \mathrm{g} / \mathrm{ml}, P>0.05$ ) (Figure 1a). There was no significant difference in the plasma concentration of C1qCIC between the KD group and healthy control group, or between the KD group and febrile control group (36.53 \pm 7.57 vs. $35.81 \pm 8.41 \mathrm{mU} / \mathrm{l}, 36.53 \pm 7.57$ vs. $36.27 \pm 8.81 \mathrm{mU} / \mathrm{l}$, all $P>0.05)$ (Figure $1 b$ ).

Mannan-binding lectin-associated serine protease (MASP-1). MASP-1 is a component of the lectin pathway of complement activation. Compared with the healthy control group, the plasma level of MASP-1 in the KD group was decreased significantly (5.25 (4.77) vs. $6.02(4.54) \mu \mathrm{g} / \mathrm{ml}, P<0.05)$. However, there was no significant difference in the plasma concentration of MASP-1 between the KD group and febrile control group (5.25 (4.77) vs. $5.94(3.42) \mu \mathrm{g} / \mathrm{ml}, P>0.05)$ (Figure 1c).

Factor B. Factor B is a component of the alternative pathway of complement activation. The plasma level of factor B was 
significantly lower in the KD group than that in healthy control and febrile control groups $(0.07 \pm 0.03$ vs. $0.17 \pm 0.06$ and $0.11 \pm 0.04 \mu \mathrm{g} / \mathrm{ml}$, all $P<0.01$ ) (Figure 1d).

C4d. C4d is a product derived from the activation of $\mathrm{C} 4$, which is needed in the classical pathway and lectin pathway. C4d is a stable product bound covalently to vascular endothelial cells. The plasma level of C4d was significantly lower in the KD group than that in the healthy control group $(0.96 \pm 0.39$ vs. $1.26 \pm 0.90$ $\mu \mathrm{g} / \mathrm{ml}, P<0.05)$. However, there was no significant difference in the plasma concentration of $\mathrm{C} 4 \mathrm{~d}$ between the $\mathrm{KD}$ group and febrile control group $(0.96 \pm 0.39$ vs. $1.13 \pm 0.60 \mu \mathrm{g} / \mathrm{ml}$, $P>0.05)$ (Figure 1e).

$C 3 d, C 5 a, C 5 b-9$, and CD59. C3d is a breakdown product of complement activation. Investigation of $\mathrm{C} 3 \mathrm{~d}$ levels may provide important information about the extent of complement activation by classical, lectin, and alternative pathways. C5a and C5b are common activators of these three pathways. CD59 regulates the action of $\mathrm{C} 5$, and may be involved in the pathogenesis of $\mathrm{KD}$.
The plasma level of C3d was significantly higher in the KD group than that in the healthy control group (2.28 \pm 1.02 vs. $1.88 \pm 1.10 \mu \mathrm{g} / \mathrm{ml}, P<0.05)$. However, there was no significant difference in the plasma concentration of C3d between the KD group and febrile control group $(2.28 \pm 1.02 \mathrm{vs} .2 .12 \pm 1.20 \mu \mathrm{g} / \mathrm{ml}$, $P>0.05)$ (Figure 1f).

The plasma level of C5a was significantly lower in the KD group than that in healthy control and febrile control groups $(0.02 \pm 0.01$ vs. $0.13 \pm 0.06 \mu \mathrm{g} / \mathrm{ml}$ and $0.13 \pm 0.07 \mu \mathrm{g} / \mathrm{ml}$, all $P<0.01$ ) (Figure 1g).

Compared with the healthy control group, the plasma level of C5b-9 in the KD group was decreased significantly $(0.29$ (0.63) vs. $0.91(1.93) \mu \mathrm{g} / \mathrm{ml}, P<0.01)$. However, there was no significant difference in the plasma concentration of C5b-9 between the KD group and febrile control group $(0.29(0.63)$ vs. $0.67(0.97) \mu \mathrm{g} / \mathrm{ml}, P>0.05)$ (Figure $1 \mathrm{~h}$ ).

There was no significant difference in the plasma concentration of CD59 in the KD group and healthy control and febrile control groups $(0.16(0.28)$ vs. $0.19(0.16)) \mu \mathrm{g} / \mathrm{ml}$, vs. 0.24 (0.15) $\mu \mathrm{g} / \mathrm{ml}$, all $P>0.05)$ (Figure 1i). a

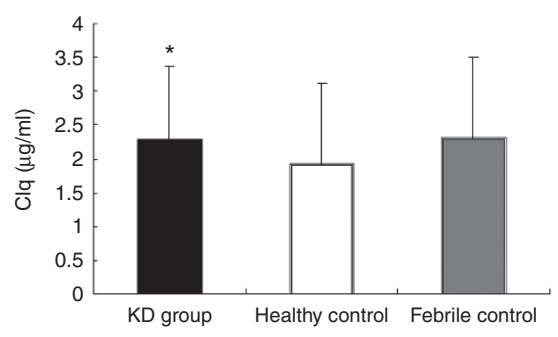

d

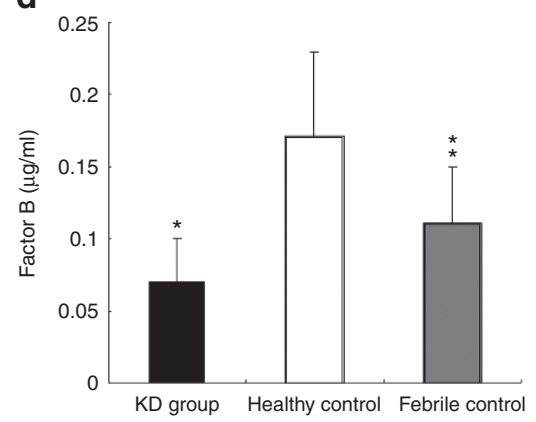

g

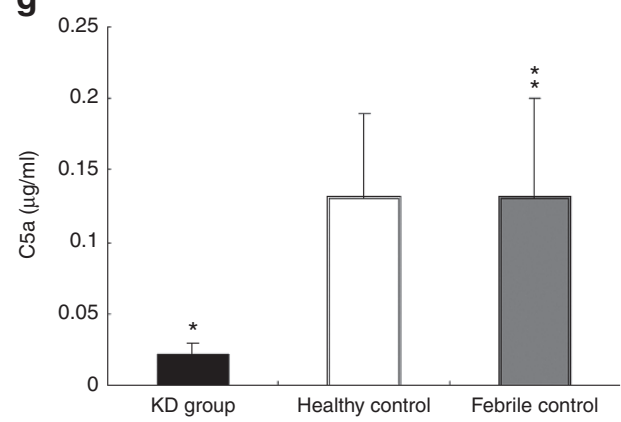

b

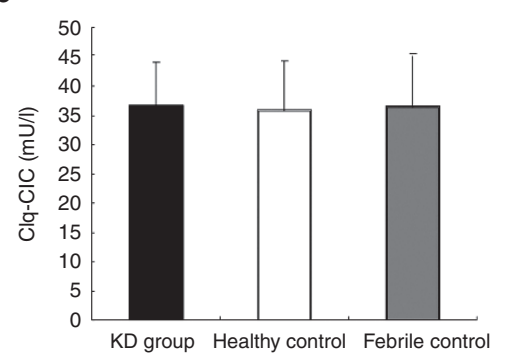

e

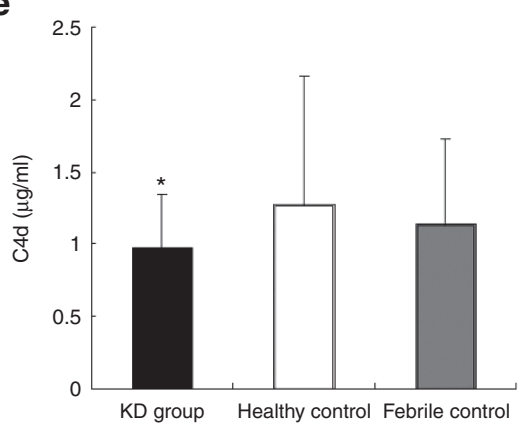

h

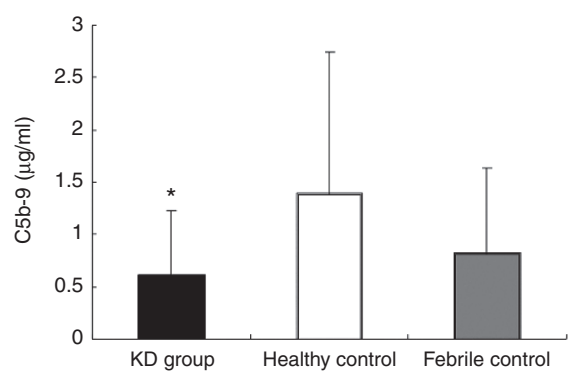

C

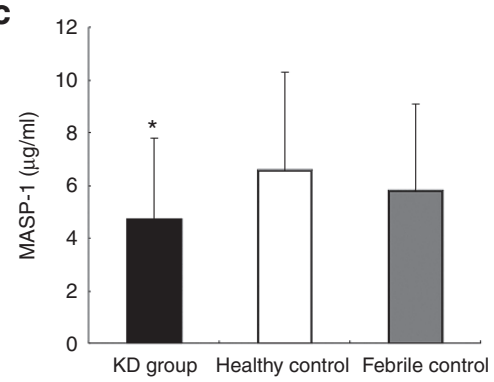

f

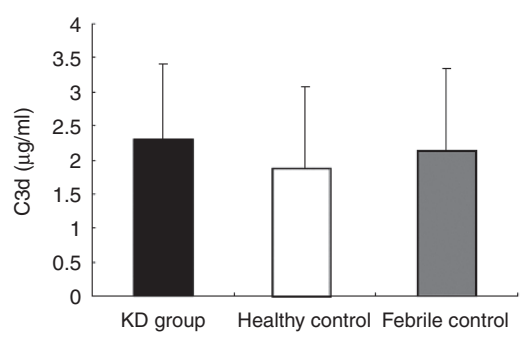

i

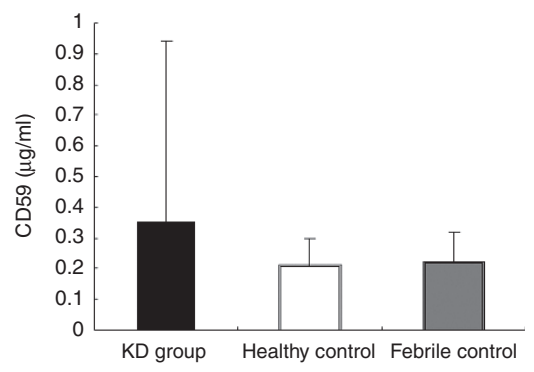

Figure 1. Plasma levels of complement components in the acute phase of KD, healthy controls and febrile controls. (a) C1q. (b) C1q-CIC. (c) MASP-1. (d) Factor B. (e) C4d. (f) C3d. (g) C5a. (h) C5b-9. (i) CD59. C1q-CIC, C1q-circulating immune complex; KD, Kawasaki disease; MASP-1, mannan-binding lectinassociated serine protease. ${ }^{*} P<0.05, \mathrm{KD}$ group vs. healthy control. ${ }^{* *} P<0.05, \mathrm{KD}$ group vs. febrile control. 
a

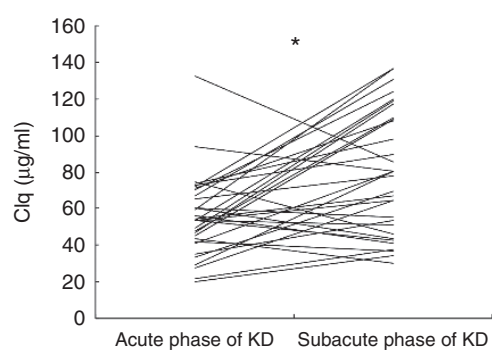

d

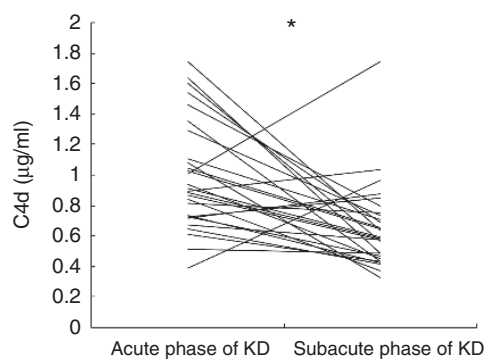

$\mathbf{g}$

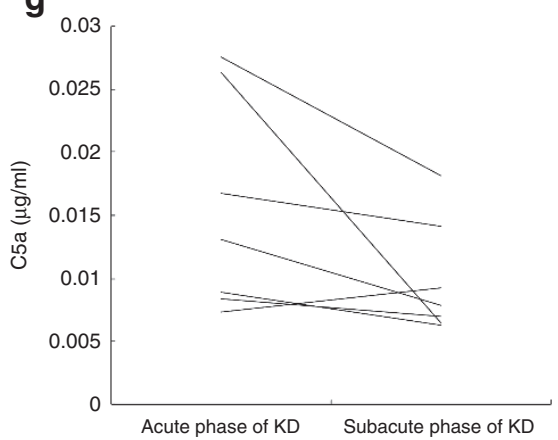

b

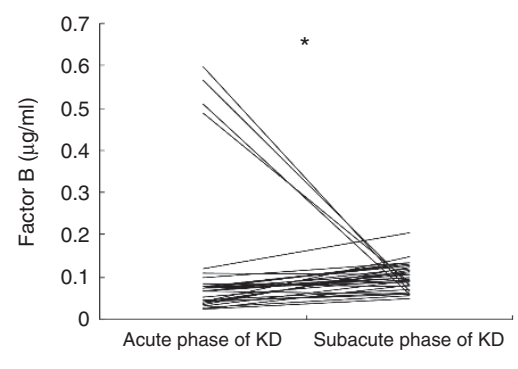

e

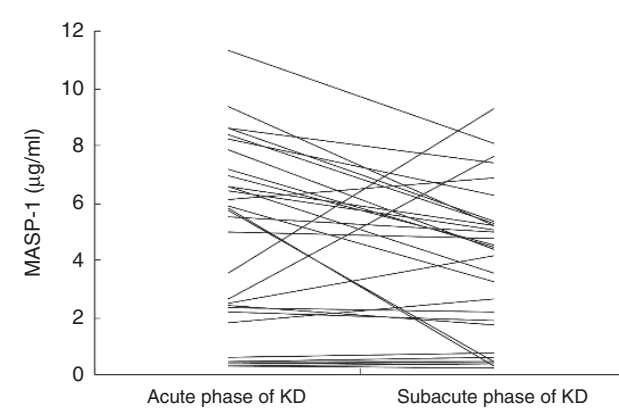

h

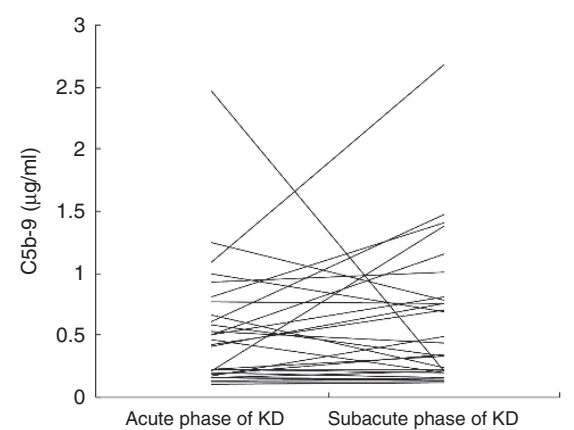

C

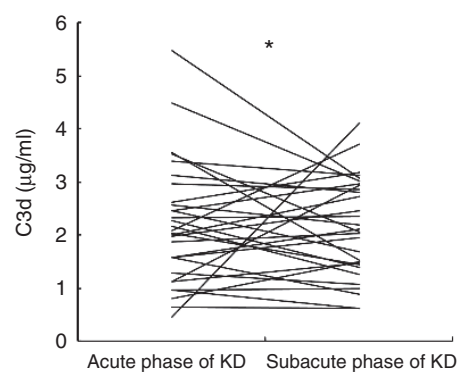

f

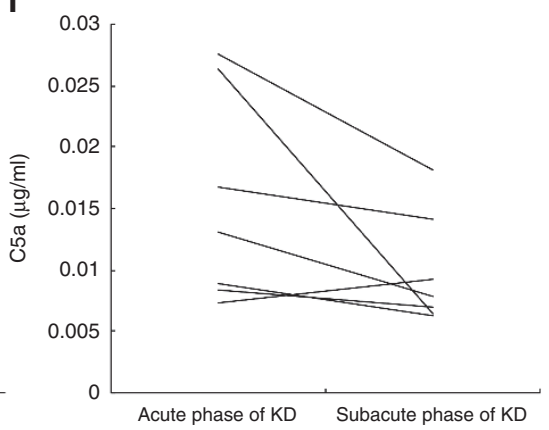

i

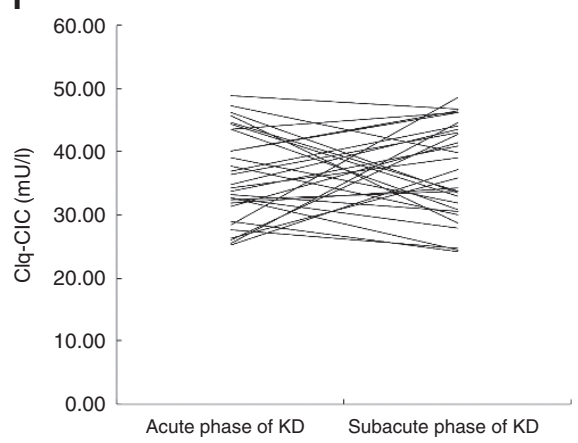

Figure 2. Measurement of changes in circulating levels of complement components in 32 patients with Kawasaki disease (KD) using sequential plasma samples. (a) C1q. (b) Factor B. (c) C3d. (d) C4d. (e) MASP-1. (f) CD59. (g) C5a. (h) C5b-9. (i) C1q-CIC. C1q-CIC, C1q-circulating immune complex; MASP-1, mannan-binding lectin-associated serine protease. ${ }^{*} P<0.05$.

\section{Changes in plasma levels of complement components in acute and subacute phases of KD}

To further confirm changes in circulating levels of complement components during KD, plasma concentrations of complement components of 32 patients in acute and subacute phases were measured.

Compared with the acute phase, the plasma concentration of $\mathrm{C} 1 \mathrm{q}$, factor B, and C3d increased significantly in the subacute phase $(79.38 \pm 33.56$ vs. $55.72 \pm 22.00 \mu \mathrm{g} / \mathrm{ml}, P<0.01$, Figure $2 \mathrm{a} ; 0.10 \pm 0.03$ vs. $0.06 \pm 0.02 \mu \mathrm{g} / \mathrm{ml}, P<0.01$, Figure $2 \mathrm{~b}$; $2.76 \pm 1.12$ vs. $2.28 \pm 1.02 \mu \mathrm{g} / \mathrm{ml}, P<0.05$, Figure $2 \mathrm{c}$ ).

Compared with the acute phase, the plasma concentration of C4d, MASP-1, and CD59 decreased significantly in the subacute phase $(0.64 \pm 0.26$ vs. $0.98 \pm 0.36 \mu \mathrm{g} / \mathrm{ml}, P<0.01$, Figure 2d; (4.27 (4.63) vs. $5.63(5.20) \mu \mathrm{g} / \mathrm{ml}, P<0.05$, Figure 2e; $0.16 \pm 0.008$ vs. $0.01 \pm 0.004 \mu \mathrm{g} / \mathrm{ml}, P<0.05$, Figure $2 \mathrm{f})$.

There were no significant differences in the plasma concentration of C5a, C5b-9, and C1q-CIC between acute and subacute phases of KD $(0.09(0.20)$ vs. $0.15(0.27)) \mu \mathrm{g} / \mathrm{ml}$, $P>0.05$, Figure 2g; $(0.33(0.63)$ vs. $0.32(0.49)) \mu \mathrm{g} / \mathrm{ml}$,
$P>0.05$, Figure 2h); $(36.53 \pm 7.57$ vs. $37.3 \pm 7.7 \mathrm{mU} / \mathrm{l}$, $P>0.05$, Figure 2i). The plasma concentration of $\mathrm{C} 5 \mathrm{a}$ of several patients in the subacute phase was below the minimum value of the reference range, which is not included in Figure 2.

Correlation between the plasma concentration of factor $\mathrm{B}, \mathrm{C} 5 \mathrm{a}$, and inflammatory markers in acute and subacute phases of KD Values of inflammatory markers in the acute phase were measured: erythrocyte sedimentation rate (ESR), 61.26 \pm $25.26 \mathrm{~mm} / \mathrm{h}$; C-reactive protein (CRP), $83.27 \pm 53.93 \mathrm{mg} / \mathrm{l}$; white blood cell (WBC) count, $16.21 \pm 5 \times 10^{9} / 1$. Values of inflammatory markers in the subacute phase were also measured: CRP $(6.1,4.7) \mathrm{mg} / \mathrm{l}$; WBC count, $8.86 \pm 2.67 \times 10^{9} / 1$.

There was no obvious correlation between plasma levels of factor B with CRP, ESR or WBC count in patients with acutephase KD (all $P>0.05$ ) (Figure $3 \mathrm{a}-\mathrm{c}$ ). There was no obvious correlation between plasma levels of C5a with CRP, ESR, and WBC count in patients with acute-phase $\mathrm{KD}($ all $P>0.05$ ) (Figure 3d-f). 


\section{Articles $\mid$ Zou et al.}

a

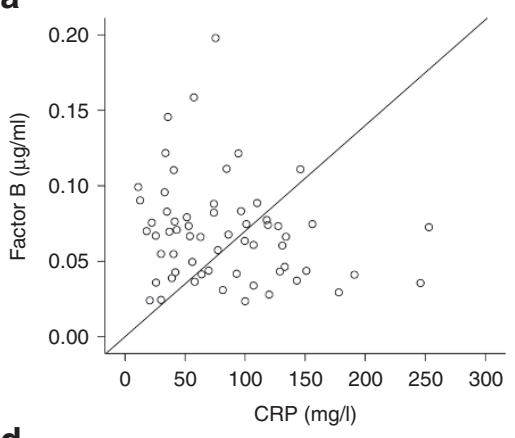

d

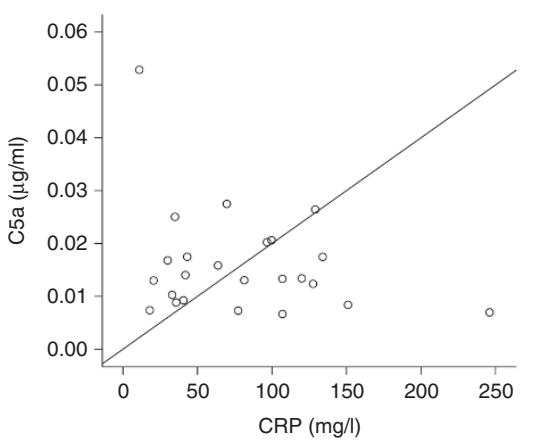

g

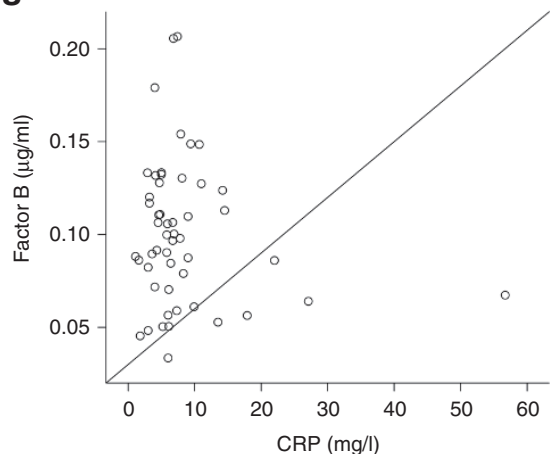

b

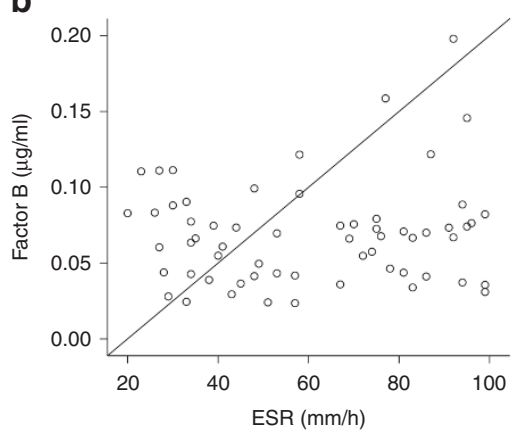

e

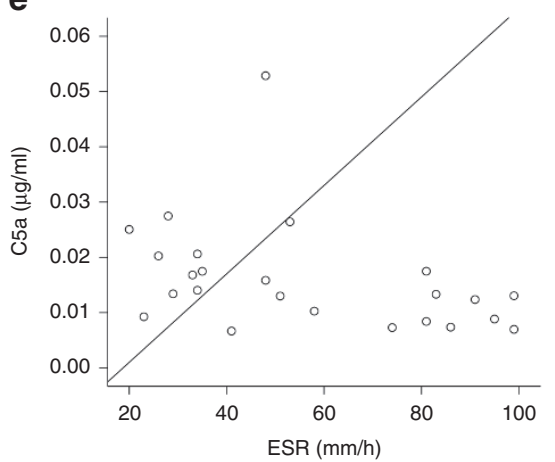

h

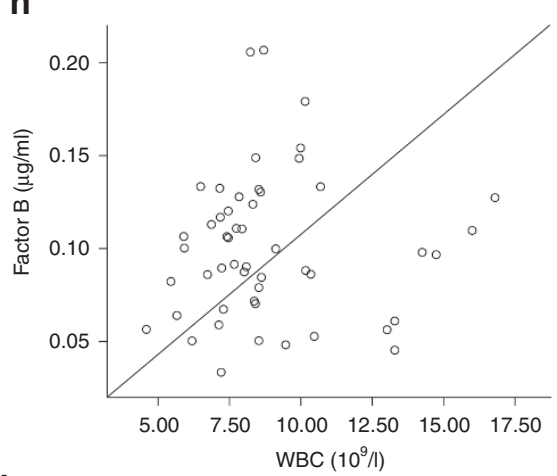

j

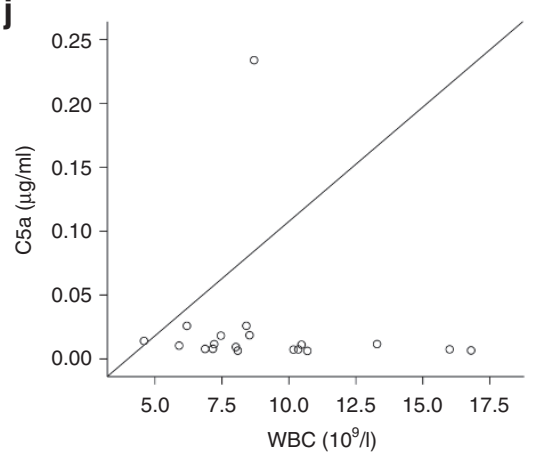

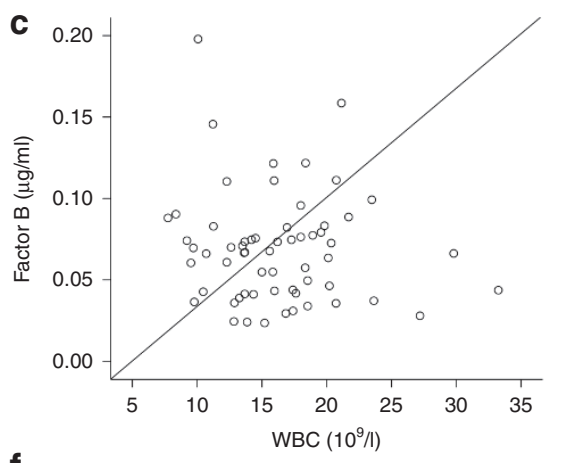

f

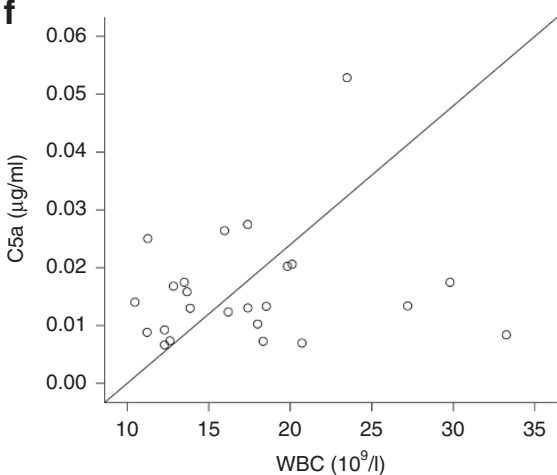

i

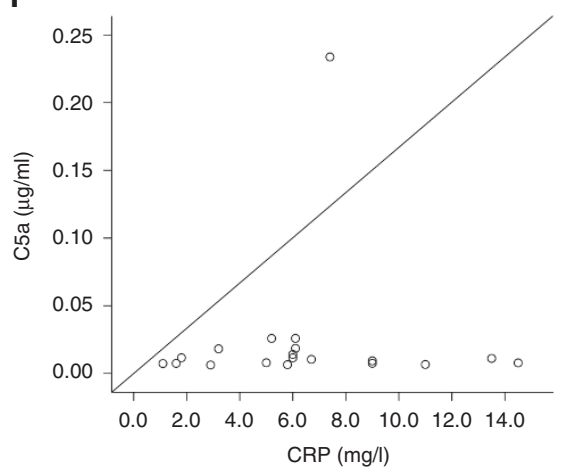

Figure 3. Correlation between the plasma concentrations of factor $B$ and $C 5$ a with inflammatory markers in acute and subacute phases of KD. (a) Association between plasma levels of factor B and C-reactive protein (CRP) in acute-phase KD $(r=-0.17, P>0.05)$. (b) Association between plasma levels of factor B and the erythrocyte sedimentation rate (ESR) in acute-phase KD $(r=0.02, P>0.05)$. (c) Association between plasma levels of factor B and total white blood cell (WBC) counts in acute-phase KD $(r=-0.11, P>0.05)$. (d) Association between plasma levels of C5a and CRP in acute-phase KD $(r=0.17$, $P>0.05)$. (e) Association between plasma levels of C5a and the ESR in acute-phase KD $(r=0.27, P>0.05)$. (f) Association between plasma levels of C5a and WBC counts in acute-phase KD $(r=0.12, P>0.05))$. (g) Association between plasma levels of factor $B$ and $C R P$ in subacute-phase KD $(r=-0.15$, $P>0.05)$. (h) Association between plasma levels of factor B and WBC count in subacute-phase KD $(r=0.04, P>0.05)$. (i) Association between plasma levels of $\mathrm{C} 5 \mathrm{a}$ and $\mathrm{CRP}$ in subacute-phase KD $(r=-0.12, P>0.05)$. (j) Association between plasma levels of $\mathrm{C} 5 \mathrm{a}$ and WBC count in subacute-phase KD $(r=-0.38$, $P>0.05)$. 
There was no obvious correlation between plasma levels of factor B with CRP and WBC count in patients with subacute KD (all $P>0.05$ ) (Figure 3g,h). There was no obvious correlation between plasma levels of C5a with CRP and WBC count in patients with subacute $\mathrm{KD}$ (all $P>0.05$ ) (Figure $3 \mathbf{i}, \mathbf{j}$ ).

\section{DISCUSSION}

The complement system is part of the innate immune system, and comprises $>30$ plasma- and membrane-bound proteins. Activation of the complement system follows three main pathways: classical, lectin, and alternative. The complement system is involved in the pathogenesis of several systemic vasculitides: antineutrophil cytoplasmic antibody-associated vasculitis; antiglomerular basement membrane disease; cryoglobulinemic vasculitis; Henoch-Schönlein purpura/immunoglobulin (Ig)A nephropathy; and KD (9).

It has been reported that the classical pathway and mannosebinding lectin pathway are activated in KD patients and experimental animal models for $\mathrm{KD}(10,11)$. However, the overall condition of the complement system (such as the activation pathway and changes in the components or fragments of the complement system) in KD is not known. Therefore, we measured various circulating components of the complement system to investigate their activation and the clinical significance of such activation in $\mathrm{KD}$ patients.

In the present study, the plasma level of $\mathrm{Clq}$ (which is activated at the onset of the classical pathway) was significantly lower in KD patients than in normal controls. This phenomenon might be due to C1q immune complex recognition sites, which result in activation of, and consumption of the components of, the classical pathway, leading to decreases in plasma levels of C1q. Studies have shown that decreased levels of C1q might be related to increased levels of C1qAb (12). Plasma levels of C1q-CIC in the present study differed from those in other reports, which might be related to the time of measurement and methods employed (13-15). The present study did not show significant differences in plasma concentrations of $\mathrm{C} 1 \mathrm{q}-\mathrm{CIC}$ between the $\mathrm{KD}$ group and healthy control or febrile control groups.

MASP-1 was the first component to be discovered in the lectin pathway of complement activation (16). Recent studies have shown that MASP- 1 is the most important protease in the lectin pathway because it can activate MASP-2 (17). We found that the plasma concentration of MASP-1 was significantly lower in the KD group than that in normal controls, which reflects lectin pathway-based activation of the complement system.

Factor $\mathrm{B}$ is an important component in the alternative complement pathway, and is also known as a precursor of C3 activation. Factor $\mathrm{B}$ is separated into two fragments ( $\mathrm{Ba}$ and $\mathrm{Bb}$ ) by factor $\mathrm{D}$. $\mathrm{Bb}$ and $\mathrm{C} 3 \mathrm{~b}$ combine to form a component of the alternative pathway, C3 convertase (18). We found that the plasma level of factor B was significantly lower in the KD group than that in normal control and febrile control groups, thereby providing evidence of activation of the alternative pathway. Our finding that the plasma concentration of factor $\mathrm{B}$ was significantly higher in the subacute phase of KD than that in the acute phase is consistent with a report (10) stating that the $\mathrm{Bb}$ level on day-10 is significantly higher than normal. However, four children had abruptly decreased levels of factor B in the subacute phase compared with the extremely high levels in the acute phase. The common feature of these children was that they were aged $<1 \mathrm{y}$. Whether age is associated with changes in plasma levels of factor B merits further research.

$\mathrm{C} 4 \mathrm{~d}$ is formed by cleavage of $\mathrm{C} 4 \mathrm{~b}$, which is generated by activation of immune complexes in classical and lectin pathways (10). A study by Murata (19) reported no significant change of C4d levels in vasculitis-type diseases and that the plasma level of $\mathrm{C} 4 \mathrm{~d}$ in the acute phase of $\mathrm{KD}$ is significantly higher than that in controls. We found that the plasma level of C4d decreased in the acute phase of KD. Nevertheless, the changing trend of the plasma level of $\mathrm{C} 4 \mathrm{~d}$ during $\mathrm{KD}$ was in accordance with the study by Murata (19), which suggests that further investigation is needed.

Activation of the complement system leads to the cleavage of C3 to C3a, C3b, C3d, C3e, and C3g. C3d is the minimum fragment of a breakdown product that cannot be cleaved by proteases. C5 convertase cleaves $\mathrm{C} 5$ protein into $\mathrm{C} 5 \mathrm{a}$ and $\mathrm{C} 5 \mathrm{~b}$. Then, C5 convertase initiates the final phase of the complement cascade (which is identical for all three pathways) and then C5b-9 is generated by assembly of C5b through to C9. We found the plasma concentration of C3d to be significantly higher in the subacute phase than that in the acute phase of $\mathrm{KD}$. This finding is consistent with reports stating the level of C3d on day-10 to be significantly higher than that in the acute phase (10). We also found the plasma concentration of

Table 1. Diagnostic criteria for Kawasaki disease as well as specific clinical data and outcome in 61 patients

\begin{tabular}{|c|c|c|}
\hline Criterion & Manifestations & Cases \\
\hline Fever & Present for $\geq 5 \mathrm{~d}$, typically high-spiking and remittent, lasting a mean of $10 \mathrm{~d}$ if untreated & 61 \\
\hline Conjunctivitis & Bilateral conjunctival injection, typically limbic sparing, nonexudative & 57 \\
\hline Lymphadenopathy & $\begin{array}{l}\text { Cervical lymphadenopathy; may be single node }>1.5 \mathrm{~cm} \text { in diameter or several smaller, firm, } \\
\text { nonfluctuant nodes bilaterally }\end{array}$ & 46 \\
\hline Extremity changes & Periungual desquamation may follow in the subacute phase & 40 \\
\hline
\end{tabular}


C5a and C5b-9 to be significantly lower in KD patients than that in normal controls. Interestingly, the plasma concentration of C5a was significantly lower in KD patients than that in febrile controls. This decrease in plasma level of C5a might have resulted from rapid combination with $\mathrm{C} 5 \mathrm{aR}$ in peripheral blood (20). It has also been postulated that decreased levels of C5a may protect against the inflammatory response (21).

CD59 may prevent formation of the MAC by blocking interactions of the C5b-8 complex with C9. CD59 can also "mop up" immune complexes and promote phagocytosis (22). We found that the CD59 level decreased in the subacute phase rather than in the acute phase of $\mathrm{KD}$. However, we did not show that the plasma concentration of C5b-9 increased in KD patients. Hence, CD59 expression in KD patients needs further investigation.

The clinical significance of the present study is that decreased plasma levels of factor B and C5a in the acute phase of KD might be used as laboratory markers for the early diagnosis of $\mathrm{KD}$. Absence of definitive diagnostic markers limits the accuracy of clinical evaluations of subjects suspected of having KD. It is a great challenge for pediatricians to diagnose the early stage of KD (especially for atypical KD with incomplete clinical manifestations). The present study showed that the concentrations of factor B and C5a in plasma were obviously lower than those in healthy controls and febrile controls in the acute phase of KD. These features can be used as biological markers to differentiate $\mathrm{KD}$ from other febrile diseases at an early stage.

We also analyzed the correlation between factor B, C5a, and other inflammatory factors (WBC, CRP, ESR): a significant correlation was not observed. Plasma levels of factor B and C5a may be indicators of immune and inflammatory responses that are independent of other inflammation-related factors in $\mathrm{KD}$.

The present study showed various complement-activation pathways in KD. In vasculitis-type diseases, in addition to liver cells, vascular endothelial cells are an important way of generating complement. Our cohort of children with KD had various liver lesions and dysfunction of endothelial cells. Either liver cells or endothelial cells (or both) had effects on generation of complement components, and this area needs further investigation. Moreover, we reported only the dynamic changes in levels of components of the complement system in acute and subacute phases of $\mathrm{KD}$; dynamic changes in the recovery stage must also be studied.

\section{Conclusion}

The present study demonstrated that there is more than one complement pathway is activated in $\mathrm{KD}$. Interactions between circulating components/fragments of the complement may increase the number and extent of inflammatory reactions and/or bloodvessel lesions in KD. Plasma concentrations of factor B and C5a were significantly lower than those in healthy and febrile controls, which might enable their use as biomarkers for the early diagnosis of $\mathrm{KD}$.

\section{METHODS}

The research program was in compliance with the Declaration of Helsinki and was approved by the Medical Ethics Committee of a Children's Hospital affiliated to the Capital Institute of Pediatrics
(Beijing, China). Informed consent was obtained from the parents of the patients.

\section{Patients}

Sixty-one consecutive patients diagnosed as having KD (according to validated diagnostic criteria (23) (Table 1) in the acute phase before initiation of intravenous immunoglobulin ( $2 \mathrm{~g} / \mathrm{kg}$ body weight) plus aspirin (ASP; $30-50 \mathrm{mg} / \mathrm{kg}$ body weight) therapy, 56 consecutive patients with $\mathrm{KD}$ in the subacute phase after the therapy described above, 32 of whom were in both acute and subacute phases, comprised the study cohort.

Patients were in a Children's Hospital affiliated to Capital Institute of Pediatrics from March 2011 to November 2013. Clinical and laboratory data were complete for all patients. Sixty-one patients with acute-phase KD had an age ranged from 3 mo to $5 \mathrm{y}$ (mean, $1.53 \pm 1.11 \mathrm{y}$ ), and the ratio of males:females was $1.25: 1$. Fifty-six patients with subacute $\mathrm{KD}$ had an age range from 2 mo to $5 \mathrm{y}$ (mean: $1.57 \pm 1.12 \mathrm{y}$ ), and the ratio of males:females was $1.67: 1$. Patients who underwent nonstandard therapy before hospitalization and who had a relevant history of cardiovascular disease were excluded from the study.

Forty age- and sex-matched healthy children undergoing health examinations and 40 patients hospitalized with febrile respiratory infectious diseases (e.g., infections of the upper respiratory tract, bronchitis, pneumonia) in the same period in our hospital comprised healthy controls and febrile controls, respectively. The age range for subjects in the healthy control group ranged from 1 y to $5 \mathrm{y}$ (mean: $3.53 \pm 1.01$ y) and the ratio of males:females was 2.08:1. The age range for subjects in the febrile control group was 7 mo to $5 \mathrm{y}$ (mean: $2.24 \pm 1.33$ y) and the ratio of males:females was 2.08:1.

\section{Collection and Processing of Blood Samples}

Blood samples from the KD group were collected before initiation of standard therapy from day- 6 to day-10 (acute phase) and after standard therapy for 3-5 d and from day-10 to day-14 (subacute phase). Samples of venous blood $(2 \mathrm{ml})$ after a morning fast were drawn and injected into sealed tubes coated with ethylenediamine tetra-acetic acid at $0.34 \mathrm{M}$ ethylenediamine tetra-acetic acid per $4.5 \mathrm{ml}$ of blood sample.

Blood samples were stored immediately at $4{ }^{\circ} \mathrm{C}$ to prevent complement degradation ex vivo. After centrifugation (3,000 rpm for $15 \mathrm{~min}$ at $\left.4{ }^{\circ} \mathrm{C}\right)$, plasma was separated $(200 \mu \mathrm{l} /$ tube $)$. Repeat freezing and thawing was avoided when testing. Samples were stored at $-80^{\circ} \mathrm{C}$ before use. Meanwhile, blood samples were collected for measurement of inflammatory markers such as CRP, total WBC count, and the ESR. Because of infusion of intravenous immunoglobulin in the acute phase, the globulin concentration in plasma increases, which leads to acceleration of the ESR. Hence, the ESR cannot accurately reflect acute-phase KD, so we did not meaure the ESR in the subacute phase. Blood samples of subjects in the healthy control group and febrile control group were collected as described above.

\section{Measurement of Complement Components in Plasma}

Plasma levels of C1q, C1q-circulating immune complex (C1q-CIC), MASP-1, factor B, C4d, C3d, C5a, C5b-9, and CD59 were measured using an enzyme-linked immunosorbent assay. Experiments were undertaken according manufacturer (USCN Life Science, Wuhan, China) instructions.

\section{Statistical Analyses}

Data analyses were undertaken using SAS v9.2 (SAS Institute, Cary, NC). Data for MASP-1, C5b-9, and C59 demonstrated non-normal distributions. They are expressed as median (four percentile interval, P75-P25) values and were analyzed by the rank-sum test. Other data had a normal distribution and are expressed as the mean \pm SD and were assessed by one-way ANOVA and the least significant difference $t$-test. The Spearman correlation was used to measure the relationship between two non-normally distributed variables, or between one non-normally distributed variable and one normally distributed variable. The Pearson correlation was used to measure the relationship between two normally distributed variables. $P<0.05$ was considered significant. 


\section{ACKNOWLEDGMENTS}

We thank the patients and their families for participating in this study.

\section{STATEMENT OF FINANCIAL SUPPORT}

No financial assistance was received to support this study.

Disclosures: There are no any financial ties to products in the study or potential/perceived conflicts of interest.

\section{REFERENCES}

1. Burns JC, Shike H, Gordon JB, Malhotra A, Schoenwetter M, Kawasaki T. Sequelae of Kawasaki disease in adolescents and young adults. J Am Coll Cardiol 1996;28:253-7.

2. Alexoudi I, Kanakis M, Kapsimali V, Vaiopoulos G. Kawasaki disease: current aspects on aetiopathogenesis and therapeutic management. Autoimmun Rev 2011;10:544-7.

3. Meri S. Complement activation in diseases presenting with thrombotic microangiopathy. Eur J Intern Med 2013;24:496-502.

4. Oksjoki R, Kovanen PT, Meri S, Pentikainen MO. Function and regulation of the complement system in cardiovascular diseases. Front Biosci 2007;12:4696-708.

5. Lewis MJ, Malik TH, Fossati-Jimack L, et al. Distinct roles for complement in glomerulonephritis and atherosclerosis revealed in mice with a combination of lupus and hyperlipidemia. Arthritis Rheum 2012;64:2707-18.

6. Haydey RP, Patarroyo de Rojas M, Gigli I. A newly described control mechanism of complement activation in patients with mixed cryoglobulinemia (cryoglobulins and complement). J Invest Dermatol 1980;74:328-32.

7. Ishikawa T, Iwashima S. Endothelial dysfunction in children within 5 years after onset of Kawasaki disease. J Pediatr 2013;163:1117-21.

8. Ando T, Nagai K, Chikada M, et al. Proteomic analyses of aortic wall in patients with abdominal aortic aneurysm. J Cardiovasc Surg (Torino) 2011;52:545-55.

9. Chimenti MS, Ballanti E, Triggianese P, et al. Vasculitides and the complement system: a comprehensive review. Clin Rev Allergy Immunol E-pub ahead of print 15 Oct 2014.

10. Kohsaka T, Abe J, Asahina T, Kobayashi N. Classical pathway complement activation in Kawasaki syndrome. J Allergy Clin Immunol 1994;93: $520-5$.

11. Nakamura A, Okigaki M, Miura N, et al. Involvement of mannose-binding lectin in the pathogenesis of Kawasaki disease-like murine vasculitis. Clin Immunol 2014;153:64-72.
12. Moroni G, Trendelenburg M, Del Papa N, et al. Anti-C1q antibodies may help in diagnosing a renal flare in lupus nephritis. Am J Kidney Dis 2001;37:490-8.

13. Salo E, Kekomäki R, Pelkonen P, Ruuskanen O, Viander M, Wagner O. Kawasaki disease: monitoring of circulating immune complexes. Eur J Pediatr 1988;147:377-80.

14. Furuse A, Matsuda I. Circulating immune complex in the mucocutaneous lymph node syndrome. Eur J Pediatr 1983;141:50-1.

15. Mason WH, Jordan SC, Sakai R, Takahashi M, Bernstein B. Circulating immune complexes in Kawasaki syndrome. Pediatr Infect Dis 1985;4: $48-51$.

16. Degn SE, Jensen L, Hansen AG, et al. Mannan-binding lectin-associated serine protease (MASP)-1 is crucial for lectin pathway activation in human serum, whereas neither MASP-1 nor MASP-3 is required for alternative pathway function. J Immunol 2012;189:3957-69.

17. Megyeri M, Makó V, Beinrohr L, et al. Complement protease MASP-1 activates human endothelial cells: PAR4 activation is a link between complement and endothelial function. J Immunol 2009;183:3409-16.

18. Matsushita M, Fujita T. Activation of the classical complement pathway by mannose-binding protein in association with a novel C1s-like serine protease. J Exp Med 1992;176:1497-502.

19. Murata K, Baldwin WM 3rd. Mechanisms of complement activation, C4d deposition, and their contribution to the pathogenesis of antibodymediated rejection. Transplant Rev (Orlando) 2009;23:139-50.

20. van der Touw W, Cravedi P, Kwan WH, Paz-Artal E, Merad M, Heeger PS. Cutting edge: Receptors for $\mathrm{C} 3 \mathrm{a}$ and $\mathrm{C} 5 \mathrm{a}$ modulate stability of alloantigenreactive induced regulatory T cells. J Immunol 2013;190:5921-5.

21. Oppermann M, Götze O. Plasma clearance of the human C5a anaphylatoxin by binding to leucocyte C5a receptors. Immunology 1994;82:516-21.

22. Gorter A, Blok VT, Haasnoot WH, Ensink NG, Daha MR, Fleuren GJ. Expression of CD46, CD55, and CD59 on renal tumor cell lines and their role in preventing complement-mediated tumor cell lysis. Lab Invest 1996;74:1039-49.

23. Newburger JW, Takahashi M, Gerber MA, et al.; Committee on Rheumatic Fever, Endocarditis, and Kawasaki Disease, Council on Cardiovascular Disease in the Young, American Heart Association. Diagnosis, treatment, and long-term management of Kawasaki disease: a statement for health professionals from the Committee on Rheumatic Fever, Endocarditis, and Kawasaki Disease, Council on Cardiovascular Disease in the Young, American Heart Association. Pediatrics 2004;114:1708-33. 